\section{Aspergilosis traqueobronquial necrotizante en paciente crítico}

\author{
MAX ANDRESEN H. ${ }^{1, a}$, CARMEN PAZ VENEGAS ${ }^{2}$, CLAUDIA VEGA ${ }^{1}$,
} CARLOS ARAVENA ${ }^{2}$, MAX ANDRESEN V. ${ }^{3, b}$

\section{Necrotizing tracheobronchial aspergillosis in an immunosuppressed patient. Report of one case}

Necrotizing tracheobronchitis due to Aspergillus spp is a rare form of invasive aspergillosis. This infection is limited to or predominant in the bronchial tree. The clinical evolution is gradual: from mild non-specific manifestations of acute tracheobronchitis to severe acute respiratory insufficiency determined by a bronchial obstruction syndrome. We report a 38 years old female with systemic lupus erythematosus treated with methylprednisolone and cyclophosphamide. She developed an invasive aspergillosis, severe respiratory failure with predominant tracheobronchial damage and upper respiratory complications.

(Rev Med Chile 2015; 143: 525-530)

Key words: Antifungal agents; Aspergillosis; Bronchitis; Bronchoscopy; Echinocandins; Lung diseases, fungal.
'Departamento de Medicina

Intensiva. Pontificia Universidad

Católica de Chile.

${ }^{2}$ Departamento de Enfermedades

Respiratorias. Pontificia

Universidad Católica de Chile.

${ }^{3}$ Facultad de Medicina Pontificia

Universidad Católica de Chile.

aMaster of Science in Critical

Care.

bAlumno de Medicina.

Recibido el 28 de agosto de 2014, aceptado el 6 de enero de 2015.

Correspondencia a:

Dr. Max Andresen $\mathrm{H}$.

Departamento de Medicina Intensiva

Facultad de Medicina Pontificia Universidad Catolica de Chile.

Marcoleta 367 - 5 Piso,

Santiago-Chile.

Fono 0560223543265

andresen@med.puc.cl
L a aspergilosis invasora es una de las complicaciones infecciosas más temidas en los pacientes inmunosuprimidos. Se presenta preferentemente en enfermos con uso crónico de glucocorticoides, trasplantados de órganos sólidos, portadores de neoplasias hematológicas o en presencia de neutropenia severa prolongada ${ }^{1-5}$. El sistema más frecuentemente comprometido es el respiratorio, con un amplio espectro de presentación que incluye desde la rinosinusitis crónica hasta el aspergiloma ${ }^{6}$.

La traqueobronquitis necrotizante debida a Aspergillus spp es una forma infrecuente de aspergilosis invasora, en la cual la infección se limita o predomina en el árbol bronquial, presentándose clínicamente de manera inespecífica, lo que lleva a retraso en el diagnóstico y tratamiento y consecuentemente a un mal pronóstico ${ }^{7}$.

Se presenta el caso clínico de una paciente inmunosuprimida que desarrolló esta severa complicación infecciosa.

\section{Caso clínico}

Mujer de 38 años con historia de 8 meses de evolución de poliartralgias, rash malar y compromiso del estado general, asociado a falla renal rápidamente progresiva. Se diagnosticó lupus eritematoso sistémico (LES) y se inició tratamiento con metilprednisolona 3 g y luego ciclosfosfamida $500 \mathrm{mg} 2$ ciclos, tras lo cual presentó pielonefritis aguda por E. coli y diarrea por Clostridium difficile, que fueron tratadas. Dado el deterioro persistente de la función renal asociado a anasarca, se inició terapia de reemplazo renal, siendo trasladada a nuestra institución para continuar tratamiento. A las $48 \mathrm{~h}$ de su ingreso, evolucionó con shock séptico severo asociado a necrosis distal de ortejos de extremidades superiores e inferiores (Figura 1) requiriendo noradrenalina hasta $5 \mathrm{u} / \mathrm{kg} / \mathrm{min}$. Dentro del estudio microbiológico no invasivo presentó hemocultivos (+) a E. coli con betalactamasas de espectro extendido (BLEE) y Klebsiella 

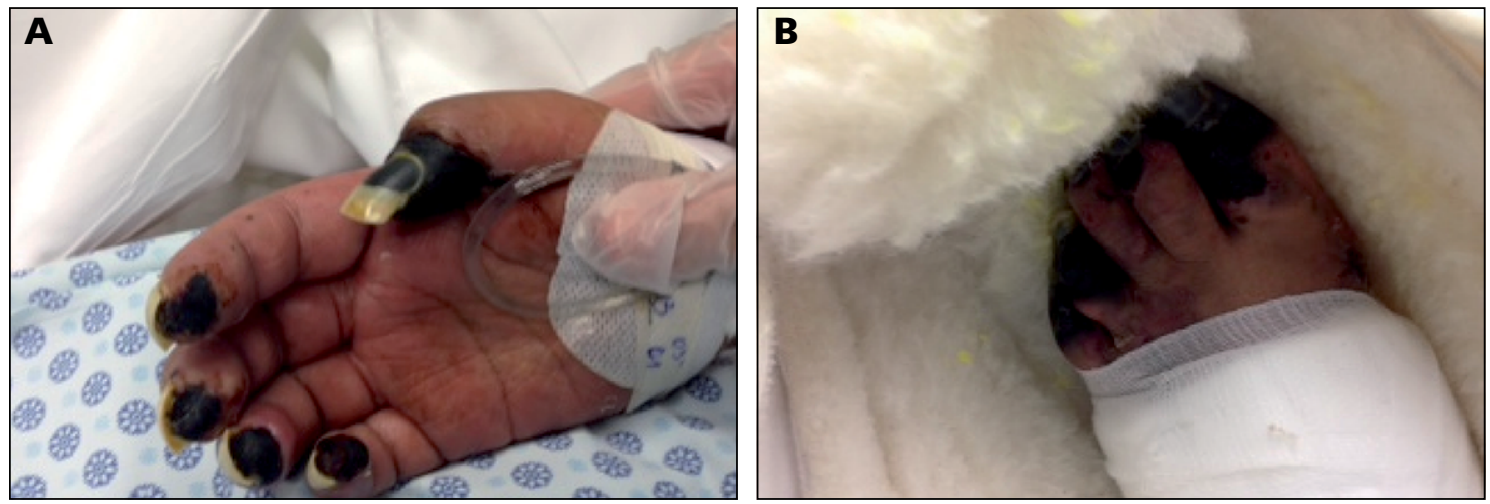

Figura 1. A y B. Se aprecia necrosis distal de ortejos en manos y pies.

pneumoniae BLEE, urocultivo (+) a Klebsiella BLEE, PCR de Citomegalovirus en sangre $(+)$ hasta 12.000 copias $/ \mathrm{mL}$, nueva diarrea por $C$. difficile con PCR en deposiciones (+). La Tomografía axial computarizada (TAC) de tórax, abdomen y pelvis reveló focos de condensación de lóbulos inferiores, leve derrame pericárdico y pleural izquierdo y pancolitis de probable etiología inflamatoria infecciosa. Evolucionó con lenta disminución de drogas vasoactivas y respuesta parcial a terapias antibióticas y antivirales.

A los 10 días reapareció fiebre, deterioro hemodinámico, elevación de parámetros inflamatorios, destacando severa obstrucción bronquial. Se realizó una nueva TAC (Figura 2) que mostró múl- tiples áreas de relleno alveolar bilateral, con opacidades parenquimatosas periféricas en densidad de "vidrio esmerilado", además de engrosamiento colónico y ascitis moderada. Se complementó estudio con fibrobroncoscopia (FBC), observándose árbol bronquial hasta nivel segmentario con mucosa eritematosa, presencia de múltiples úlceras con fondo fibrinoso y de tejido extremadamente friable (Figura 3). Se realizó lavado broncoalveolar (LBA) obteniendo una tinción de calcoflúor $(+)$ a hifas septadas, cultivo (+) a Aspergillus fumigatus y galactomanana con índice de 10,1, con valor plasmático en 9 . Se inició caspofungina y voriconazol ajustados por función renal.

La paciente evolucionó con falla respiratoria
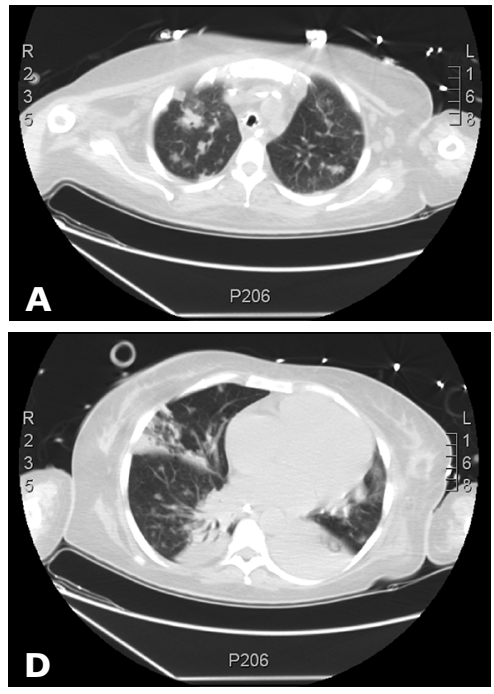
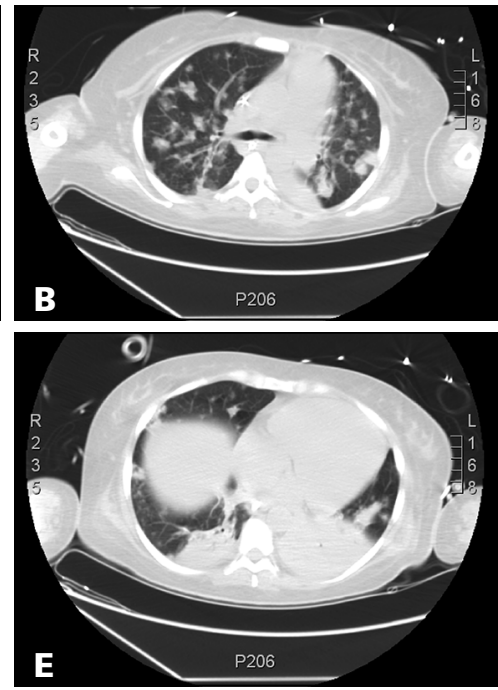

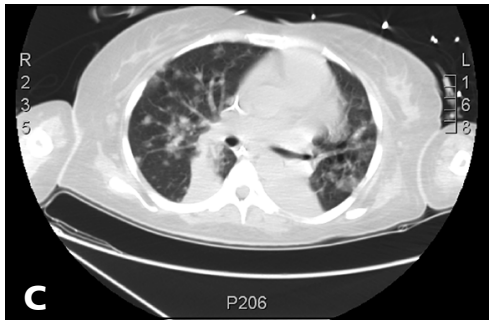

Figura 2. TAC de Tórax. Se observan múltiples áreas de relleno alveolar bilateral, con opacidades parenquimatosas periféricas en densidad de "vidrio esmerilado". 


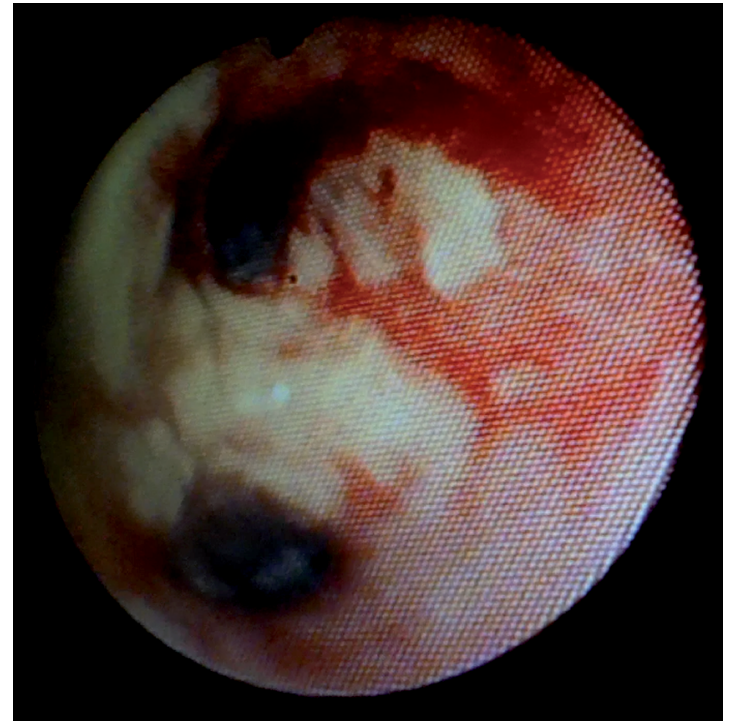

Figura 3. Fibrobroncoscopia. Se observa mucosa de bronquio fuente derecho (carina interlobar) con mucosa eritematosa y múltiples úlceras blanquecinas con fondo fibrinoso.

secundaria a severa obstrucción bronquial, requiriendo nuevas FBC de control y aseo, en las cuales se observó persistencia del grave compromiso bronquial con desprendimiento de mucosa hacia el espacio endoluminal. Se realizó una biopsia endobronquial que mostró tejido con extensa necrosis y exudado fibrinoso, con tinciones de PAS y Grocott compatibles con elementos levaduriformes y filamentosos. Dado la sostenida alteración de pruebas hepáticas con patrón colestásico, se sospechó reacción adversa a medicamentos y se decidió cambio de voriconazol a anfotericina liposomal a los 15 días de tratamiento. Evolucionó posteriormente en forma tórpida, con inestabilidad hemodinámica y nuevas intercurrencias infecciosas a pesar de amplia cobertura antibiótica, anfúngica y antiviral, falleciendo a los 55 días de su ingreso a nuestra institución.

\section{Discusión}

Aspergillus spp es un hongo filamentoso de amplia distribución en el ambiente. Aunque se han identificado más de 250 especies, sólo una minoría de ellos están asociados con enfermedades humanas, siendo Aspergillus fumigatus el patógeno más frecuente (50-60\%) de todas las infecciones por este hongo.
La aspergilosis pulmonar invasora es una de las infecciones fúngicas más comunes en huéspedes inmunocomprometidos, pudiendo comprometer el tracto respiratorio en $90 \%$ de los casos, principalmente el parénquima pulmonar y rara vez el árbol traqueobronquial $(7 \% \text { de los casos })^{8}$. La progresión desde la colonización hasta la invasión de tejido y el tipo de enfermedad que los pacientes desarrollan, dependen principalmente de su estado inmune y de los mecanismos de defensa locales ${ }^{7}$.

Datos recientes indican que la aspergilosis invasiva debe ser considerada como una enfermedad infecciosa emergente en la unidad de cuidados intensivos (UCI), incluso en ausencia de una inmunodeficiencia predisponente aparente. Un estudio realizado en una UCI médica, mostró que la incidencia de aspergilosis invasiva fue de $5,8 \%$. La mayoría de estos pacientes no tenían antecedentes de neoplasia hematológica ${ }^{9}$. Factores de riesgo independiente de mortalidad han sido: ingreso previo en una unidad de hospitalización, antecedente de inmunosupresión y presencia de sepsis grave o shock séptico ${ }^{6}$.

La primera descripción de traqueobronquitis por Aspergillus fue hecha por Wheaton et al en 1890, en una niña de dos años que falleció de neumonía ${ }^{10}$. En 1991, Kramer et $a^{11}$, describieron formas invasivas de traqueobronquitis por Aspergillus en autopsia de pacientes trasplantados de pulmón. Se dividieron los casos en dos tipos: invasivas y no invasivas. La invasiva se subdividió en tres formas morfológicas: traqueobronquitis, traqueobronquitis ulcerativa y traquebronquitis pseudomembranosa. Por su parte la no invasiva se subdividió en dos formas morfológicas: impactación mucoide y la aspergilosis obstructiva.

Denning el año $1995^{12}$, propuso una clasificación y una terminología unificada que consta de tres tipos de traqueobronquitis: a) obstructiva, que se caracteriza por crecimiento endoluminal del Aspergillus asociado a tapones mucosos gruesos y atelectasias; b) ulcerativa, que se caracteriza por formación de úlceras que penetran la pared bronquial y forman fistulas bronco esofágicas o broncoarteriales, las que pueden producir hemorragias fatales y c) pseudomembranosa, caracterizada por la formación de extensas pseudomembranas blanquecinas compuestas por hifas, fibrina y tejido necrótico. Estas distintas variantes son probablemente distintos estadios en el desarrollo de la enfermedad ${ }^{13}$. 
La mayor parte de los casos de aspergilosis traqueobronquial invasora reportados en la literatura se han observado en pacientes severamente inmunocomprometidos ${ }^{13}$, tanto trasplantados de órgano sólido ${ }^{1,14,15}$ como de médula ósea ${ }^{2,3}$, además de pacientes con $\mathrm{VIH}^{5,16}$, neutropenia o tumores hematológicos ${ }^{17,18}$. Sin embargo, también se han descrito en pacientes menos inmunocomprometidos como pacientes con lupus eritematoso sistémico sin tratamiento inmunomodulador, diabéticos, enfermedad pulmonar obstructiva crónica, cirrosis hepática avanzada, usuarios tratamiento corticoidal o inclusive en ausencia de una inmunodeficiencia predisponente aparente $^{19-24}$. El Aspergillus fumigatus es el organismo más frecuente seguido en frecuencia por A. flavus, $A$. niger y $A$. nidulans ${ }^{7}$.

Respecto a las manifestaciones clínicas de la aspergilosis traqueobronquial, pueden confundirse o diferir de la aspergilosis pulmonar invasora. Hay síntomas inespecíficos con escasos o nulos hallazgos en exámenes radiológicos, lo que produce un retraso en el diagnóstico de la enfermedad. La presencia de tos, disnea, sibilancias o hemoptisis en el contexto clínico correspondiente debe hacernos sospechar esta patología. Si bien hay poca documentación de los hallazgos radiológicos, se han descrito alteraciones bronquiales en TAC de tórax y PET CT alterados con aumento de la captación del radio trazador. El diagnóstico es broncoscópico, utilizando la visión directa y muestras por lavado o biopsias para obtención de tinciones y cultivos, lo que hace el diagnóstico definitivo. Tal como en el caso presentado, en ocasiones pueden coexistir el compromiso parenquimatoso y el de las vías respiratorias, siendo la predominancia del daño traqueobronquial el distintivo que marca la presencia de esta patología.

El tratamiento de la aspergilosis invasiva puede llegar a ser subóptimo y hay estudios dirigidos al tratamiento específico de las formas tranqueobronquiales. El voriconazol es considerado como el fármaco de elección con una tasa de sobrevida exitosa significativamente mayor en comparación con anfotericina $\mathrm{B}$ deoxicolato ${ }^{25}$. La caspofungina es actualmente la única equinocandina aprobada por la FDA para el tratamiento de la aspergilosis. El posaconazol, un nuevo triazol de amplio espectro, se ha empleado con éxito como tratamiento de rescate para los pacientes refractarios o intolerantes a la terapia convencional ${ }^{26}$. En el caso de la asper- gilosis traqueobronquial invasora se ha descrito el uso de itraconazol, anfotericina en nebulización, anfotericina B liposomal, y voriconazol con un tratamiento promedio de 25 días ( 8 a 47 días). Varios autores sugieren como tratamiento de elección a la anfotericina B deoxicolato. Se ha descrito que la combinación con caspofungina podría ser más efectiva. En la actualidad las opciones terapéuticas más usadas son las combinaciones de anfotericina más caspofungina, anfotericina con voriconazol o la monoterapia con voriconazol ${ }^{27,28}$.

El aseo bronquial mediante fibrobroscopia, realizado en esta paciente, parece ser eficaz en la eliminación de los tapones de mucus y pseudomembranas, lo que permite revertir los episodios de obstrucción de vía aérea superior, la generación de mecanismos de válvula, y por ende atrapamiento de volumen y auto PEEP. La eliminación de las masas de micelios puede ser útil debido a la escasa penetración de los antifúngicos en las masas de hongos ${ }^{29}$, pero puede aumentar el riesgo de complicaciones hemorrágicas ya que se podrían producir hemorragias fatales al eliminar pseudomembranas necrotizantes que se han extendido a través de la pared bronquial hacia vasos pulmonares ${ }^{18}$. Debemos enfatizar por tanto la broncoscopia a manos de personal experto, tanto en el diagnóstico como en el tratamiento de esta grave patología.

Debido al riesgo de provocar hemorragias letales durante la FBC, se ha sugerido realizar los procedimientos bajo ultrasonografía endobronquial (EBUS) para guiar la toma de muestras ${ }^{30}$.

En relación al pronóstico este depende de la enfermedad subyacente, del estado inmunológico y de la recuperación de la inmunosupresión, la mortalidad descrita en relación al análisis de los casos reportados supera $70 \%$ en pacientes severamente inmunocomprometidos con neoplasias hematológicas, siendo aparentemente mejor en pacientes sin estas características ${ }^{7}$.

\section{Conclusión}

La aspergilosis invasora es una infección oportunista cada vez más frecuente de observar en las unidades de paciente crítico, especialmente en aquellos pacientes con inmunosupresión marcada. Dentro de sus variadas formas de presentación, la aspergilosis traqueobronquial necrotizante es 
una de las más dramáticas, tanto por su tórpida evolución como por la dificultad que presenta su manejo. Se presentó el caso de una paciente con importantes factores de riesgo tanto de adquisición de la enfermedad como de mortalidad, con desenlace fatal pese a terapia óptima recibida.

\section{Referencias}

1. García-Gallo CL, García-Fadul C, Laporta-Hernández R, Ussetti-Gil P. Aspergillus tracheobronchitis in a lung transplant recipient. Rev Iberoam Micol 2011; 28 (3): 129-33.

2. Angelucci E, Ugolini M, Lucarelli G, Raspugli M, Baronciani D, Galimberti M, et al. Endobronchial aspergillosis in marrow transplant patients. Bone Marrow Transplant 1991; 8 (4): 328-9.

3. Koh LP, Goh YT, Linn YC, Hwang J, Tan P. Pseudomembranous tracheobronchitis caused by Aspergillus in a patient after peripheral blood stem cell transplantation. Ann Acad Med Singapore 2000; 29 (4): 531-3.

4. Denning DW, Follansbee SE, Scolaro M, Norris S, Edelstein H, Stevens DA. Pulmonary aspergillosis in the acquired immunodeficiency syndrome. N Engl J Med 1991; 324 (10): 654-62.

5. Kemper CA, Hostetler JS, Follansbee SE, Ruane P, Covington $\mathrm{D}$, Leong $\mathrm{SS}$, et al. Ulcerative and plaque-like tracheobronchitis due to infection with Aspergillus in patients with AIDS. Clin Infect Dis 1993; 17 (3): 344-52.

6. Álvarez Lerma F, Olaechea Astigarraga P, Palomar Martínez M, Rodríguez Carvajal M, Machado Casas JF, Jiménez Quintana MM, et al. Grupo de estudio ENVIN-HELICS Respiratory infections caused by Aspergillus spp. in critically ill patients admitted to the intensive care units. Med Intensiva 2014 Apr 5. pii: S0210-5691(14)00045.

7. Krenke R, Grabczak EM. Tracheobronchial manifestations of Aspergillus infections. Scientific World Journal 2011; 11: 2310-29.

8. Mylonakis E, Barlam TF, Flanigan T, Rich JD. Pulmonary aspergillosis and invasive disease in AIDS: review of 342 cases. Chest 1998; 114 (1): 251-62.

9. Meersseman W, Vandecasteele SJ, Wilmer A, Verbeken E, Peetermans WE, Van Wijngaerden E. Invasive aspergillosis in critically ill patients without malignancy. Am J Respir Crit Care Med 2004; 170: 621-5.

10. Wheaton SW. Case primarily of tubercle in which a fungus (aspergillus) grew in the bronchi and and lung, simulating actinomycosis. Trans Path Soc (Lond) 1890; 41: 34-7.
11. Kramer MR, Denning DW, Marshall SE, Ross DJ, Berry $\mathrm{G}$, Lewiston NJ, et al. Ulcerative tracheobronchitis after lung transplantation. A new form of invasive aspergillosis. Am Rev Respir Dis 1991; 144 (3 Pt 1): 552-6.

12. Denning DW Review Commentary: unusual manifestations of aspergillosis. Denning DW Thorax 1995; 50 (7): 812-3.

13. Wu N, Huang Y, Li Q, Bai C, Huang HD, Yao XP. Isolated invasive Aspergillus tracheobronchitis: a clinical study of 19 cases. Clin Microbiol Infect 2010; 16 (6): 689-95.

14. Ramos A, Segovia J, Gómez-Bueno M, Salas C, Lázaro MT, Sánchez I, et al. Pseudomembranous Aspergillus tracheobronchitis in a heart transplant recipient. Transpl Infect Dis 2010; 12 (1): 60-3.

15. Sayiner A, Kürşat S, Töz H, Duman S, Onal B, Tümbay E. Pseudomembranous necrotizing bronchial aspergillosis in a renal transplant recipient. Nephrol Dial Transplant 1999; 14 (7): 1784-5.

16. Denning DW, Follansbee SE, Scolaro M, Norris S, Edelstein $\mathrm{H}$, Stevens DA. Pulmonary aspergillosis in the acquired immunodeficiency syndrome. N Engl J Med 1991; 324 (10): 654-62.

17. Berlinger NT, Freeman TJ. Acute airway obstruction due to necrotizing tracheobronchial aspergillosis in immunocompromised patients: a new clinical entity. Ann Otol Rhinol Laryngol 1989; 98 (9): 718-20.

18. Ahn MI, Park SH, Kim JA, Kwon MS, Park YH. Pseudomembranous necrotizing bronchial aspergillosis. Br J Radiol 2000; 73 (865): 73-5.

19. Jung SW, Kim MW, Cho SK, Kim HU, Lee DC, Yoon BK, et al. A Case of Endobronchial Aspergilloma Associated with Foreign Body in Immunocompetent Patient without Underlying Lung Disease. Tuberc Respir Dis (Seoul) 2013; 74 (5): 231-4.

20. Oh HJ, Kim HR, Hwang KE, Kim SY, Ahn SH, Yang SH, et al. Case of Pseudomembranous Necrotizin Tracheobronchial Aspergillosis in an Immunocompetent Host. Korean J Intern Med 2006; 21: 279-82.

21. Cha SI, Shin KM, Yoo SS, Jeong JY, Yoon GS, Lee SY, et al. Pseudomembranous Aspergillus tracheobronchitis in an immunocompetent patient. Tuberc Respir Dis 2008; 65: 400-4.

22. Mohan A, Guleria R, Mukhopadhyaya S, Das C, Nayak A, Sharma SK. Invasive tracheobronchial aspergillosis in an immunocompetent person. American Journal of the Medical Sciences Am J Med Sci 2005; 329 (2): 107-9.

23. Sada M, Saraya T, Tanaka Y, Sato S, Wakayama M, Shibuya $\mathrm{K}$, et al. Invasive tracheobronchial aspergillosis in a patient with systemic lupus erythematosus-dermatomyositis overlap syndrome. Intern Med 2013; 52: 2149-53. 
24. Hines DW, Haber MH, Yaremko L, Britton C, Mc Lawhon RW, Harris AA. Pseudomembranous tracheobronchitis caused by Aspergillus. Am Rev Respir Dis 1991; 143 (6): 1408-11.

25. De Pauw B, Walsh TJ, Donnelly JP, Stevens DA, Edwards JE, Calandra T, et al. Revised definitions of invasive fungal disease from the European Organization for Research and Treatment of Cancer/Invasive Fungal Infections Cooperative Group and the National Institute of Allergy and Infectious Diseases Mycoses Study Group (EORTC/MSG) Consensus Group. Clin Infect Dis 2008; 46 (12): 1813-21.

26. Tasci S, Glasmacher A, Lentini S, Tschubel K, Ewig S, Molitor E, et al. Pseudomembranous and obstructive Aspergillus tracheobronchitis-optimal diagnostic strategy and outcome. Mycoses 2006; 49 (1): 37-42.

27. Walsh TJ, Anaissie EJ, Denning DW, Herbrecht R,
Kontoyiannis DP, Marr KA, et al. Treatment of aspergillosis: clinical practice guidelines of the infectious diseases society of America. Clin Infect Dis 2008; 46 (3): 327-60.

28. Karthaus M. Prophylaxis and treatment of invasive aspergillosis with voriconazole, posaconazole and caspofungina-review of the literature. Eur J Med Res 2011; 16 (4): 145-52.

29. Seidler MJ, Salvenmoser S, Müller FM. Aspergillus fumigatus forms biofilms with reduced antifungal drug susceptibility on bronchial epithelial cells. Antimicrob Agents Chemother 2008; 52 (11): 4130-6.

30. Casal RF, Adachi R, Jiménez CA, Sarkiss M, Morice RC, Eapen GA. Diagnosis of invasive Aspergillus tracheobronchitis facilitated by endobronchial ultrasound-guided transbronchial needle aspiration: a case report. J Med Case Rep 2009; 3: 9290. 